\title{
Les centres nucléaires et le public : communication, information
}

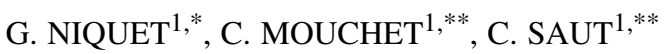

(Manuscrit reçu le 29 septembre 2004, accepté le 2 octobre 2004)

RÉSUMÉ Les CLI ont un rôle important pour suivre l'impact des centres nucléaires et communiquer les informations aux populations. L'ANCLI regroupe des CLI pour permettre une mise en commun des travaux et une bonne représentation des CLI. Les publications, l'organisation d'un colloque et des sessions de formation sont des actions de l'ANCLI. Ces ensembles CLI et ANCLI sont soumis à des textes législatifs ou réglementaires qui imposent des évolutions.

ABSTRACT The nuclear centers and public: communication, information.

CLI have an important role to check nuclear plants environmental impact and communicate informations to inhabitants. ANCLI assembles some of the CLI to exchange experiences and works and gives them a national representation. Publications, national annual symposium and learning sessions are some of his actions. CLI and ANCLI are ruled by specific legislative texts and need to adapt with them changes.

\section{Les CLI}

Les sites nucléaires $\mathrm{CNPE}^{2}$, centres de recherche ou industriels sont toujours des lieux suspects pour le public par rapport au danger et à la pollution de l'environnement. Ce risque réel ou supposé est certainement déterminé par l'attitude irrationnelle du public relativement aux rayonnements ionisants. L'absence de sensation, odeurs ou effets immédiats, associée aux souvenirs difficiles comme explosions de bombes ou l'accident de Tchernobyl peut être à l'origine de la position du public.

Cette attitude est en général amplifiée par le manque de communication ou par la diffusion d'informations parcellaires de la part des responsables des centres nucléaires. Comme on peut le concevoir, il est très difficile d'obtenir des informations fiables et complètes.

ANCLI, c/o CLI du Gard/ DDEAT, Hôtel du département, rue Guillemette, 34000 Nîmes, France.

Président de l'ANCLI.

** Chargées de mission à l'ANCLI.

2 CNPE, Centre nucléaire de production d'électricité. 
Ainsi pour permettre une meilleure communication et surveiller l'impact des centres nucléaires, les $\mathrm{CLI}^{3}$ ont été créées par une circulaire de Pierre Mauroy en décembre 1981 (CI, 1981). Elles ont été mises en place autour des centres nucléaires civils. Avant cette date, certains sites étaient dotés de structures spéciales pour l'information et la surveillance comme la $\mathrm{CSPI}^{4}$ de la Hague, la $\mathrm{CLS}^{5}$ de Fessenheim ou la CLE $^{6}$ de Romans-sur-Isère.

Pour les sites nucléaires intéressant la Défense des initiatives locales ont permis la création de structures analogues aux CLI, mais il faut attendre 2001 pour qu'un décret ${ }^{7}$ crée des commissions d'information. Un arrêté particulier doit définir pour chaque site la CI ainsi créée. Nous reviendrons sur ce point.

En France, dans le domaine nucléaire, il existe 30 CLI ou structures assimilées réparties comme suit :

- 18 centres de production d'électricité ;

- 2 centres de stockage ;

- 1 centre de retraitement ;

- 5 centres CEA dont 3 CEA/DAM, concernés par le décret de juillet 2001 dont nous reparlerons ;

- 2 ensembles industriels SOMANU à Maubeuge et FBFC à Romans-sur-Isère, correspondant au champ d'application de la loi sur les établissements industriels à risque ;

- 1 centre en démantèlement (Creys-Malville) ;

- 1 laboratoire de recherche pour le stockage souterrain de déchets radioactifs à vie longue (Bure).

La mission et le rôle de ces structures sont de suivre et communiquer sur l'impact des centres nucléaires. La radioprotection étant l'ensemble des moyens utilisés pour assurer la protection contre les rayonnements ionisants, les CLI font de la radioprotection au quotidien.

En général, elles sont placées auprès du Conseil général des départements d'implantation des centrales nucléaires et le Président est désigné par le Président du Conseil général. La composition de ces commissions reflète la société avec des représentants des élus, des administrations intéressées par le centre nucléaire, des associations concernées par la protection de l'environnement, des unions syndicales et de l'exploitant.

\footnotetext{
CLI, Commission locale d'information.

4 CSPI, Commission spéciale permanente d'information auprès de l'établissement COGEMA de La Hague.

CLS, Commission locale de surveillance auprès du CNPE de Fessenheim.

6 CLE, Commission locale d'environnement auprès de l'usine FBFC de Romans-sur-Isère.

Décret 2001-592 du 5 juillet 2001 relatif à la sûreté et à la radioprotection des installations et activités nucléaires intéressant la défense.
} 
L'organe des CLI utilisé pour diffuser les informations est en général un journal ou une lettre d'information distribué gratuitement aux populations situées au voisinage des centres nucléaires.

La CLI est un lieu de débat et de dialogue où au cours des réunions plénières tous les membres peuvent s'exprimer et confronter des idées et interpeller les exploitants. Cet aspect du rôle des CLI est important, en effet, le dialogue est souvent à l'origine d'une démarche pédagogique permettant une bonne compréhension entre les parties.

Les CLI sont aussi régulièrement informées par les exploitants, la DGSNR ${ }^{8}$ ou les préfectures des incidents survenus sur les centres nucléaires. Ainsi, les présidents des CLI concernées ont été sollicités lors de la canicule de 2003 lorsque la forte chaleur et le faible niveau des rivières rendant les refroidissements difficiles avaient nécessité des autorisations dérogatoires pour permettre le fonctionnement de CNPE.

Les travaux de ces commissions ne se limitent pas à faciliter des dialogues, mais consistent aussi à publier les résultats d'expertises ou de mesures effectuées dans l'environnement autour des centres nucléaires. Le stockage et l'entreposage des déchets nucléaires sont également des préoccupations des CLI. L'importance de ces travaux est fonction du budget disponible.

Le budget des CLI est un élément difficile à dominer car il est déterminé en général par le Conseil général, cependant la DGSNR contribue aux dépenses pour des expertises ou des actions exceptionnelles. À titre d'exemple, on peut rappeler que l'ensemble des CLI de France dispose d'un budget de l'ordre de 680000 euros dont environ la moitié provient de la DGSNR.

Les expertises effectuées par les CLI portent sur le marquage par des éléments radioactifs des constituants de la chaîne alimentaire ou de l'environnement, eaux stagnantes ou des rivières, herbes, ... Ces résultats sont en règle générale identiques, compte tenu des erreurs expérimentales, à ceux obtenus par les exploitants. L'interprétation de ces mesures peut être objet de discussion. Il faut rappeler que la référence aux normes pour la dose limite de $1 \mathrm{mSv}$ par an (Euratom, 1996 ; Métivier, 1997) pour le public ne constitue pas une garantie sanitaire d'où les possibilités de controverse.

Les CLI travaillent dans le voisinage d'un centre nucléaire et ont peu ou pas de contact avec les autres structures ce qui est regrettable pour valoriser les actions,

8 DGSNR, Direction générale de la sûreté nucléaire et de la radioprotection. 
une étude effectuée à Fessenheim peut être pertinente pour le voisinage de Golfech. Pour permettre une mise en relation des présidents de CLI, des exploitants et le gouvernement, la DGSNR organise chaque année la conférence des Présidents, prévue par la circulaire de 1981. Le 8 décembre 2004 aura lieu la $16^{\mathrm{e}}$ conférence, le thème sera les études épidémiologiques, pour la $15^{\mathrm{e}}$ conférence, le thème était la radioprotection des travailleurs. Au cours de cette journée, le thème est décliné par plusieurs intervenants et les représentants des CLI peuvent apporter leur contribution.

\section{L'ANCLI}

Les CLI ont peu de relations, la communication inter CLI est inexistante. Ce constat a conduit des présidents de CLI à mettre en place l'ANCLI ${ }^{9}$ en septembre 2000. L'ANCLI est dirigée par un Conseil d'administration composé de 9 Présidents élus par l'Assemblée générale.

Aujourd'hui, elle regroupe 15 CLI. Les CLI participant à l'ANCLI sont volontaires et versent une cotisation permettant d'alimenter en partie le budget, l'autre partie du budget est obtenue par une subvention de la DGSNR.

Dans l'indépendance et la transparence le rôle et les objectifs de l'ANCLI sont :

- favoriser l'échange d'expérience et la mise en commun d'informations entre les structures participant à l'ANCLI ;

- définir une représentation des CLI et assurer des relations avec les organismes nationaux et internationaux ;

- offrir un soutien logistique aux CLI et faire procéder à des expertises sur tout sujet lié à la protection de l'environnement et à la sûreté nucléaire.

Le budget actuel de l'ordre de 70000 euros par an, trop faible pour donner à l'ANCLI les moyens de réaliser tous ses objectifs lui permet cependant de réaliser des actions et activités dont voici les principales :

- organisation des visites des grands centres nucléaires pour les membres des CLI ;

- présentation d'un colloque annuel sur des thèmes importants pour les CLI : septembre 2001 à Nogent-sur-Seine «la communication dans la crise », septembre 2002 à Colmar « expertises et contre-expertises », septembre 2003 à Golfech « Les CLI, les exploitants et les autres... », septembre 2004 à Dieppe « Nucléaire et développement durable ». Le colloque de septembre 2005 est prévu à Dijon avec le thème « Sûreté et sécurité »;

\footnotetext{
9 ANCLI, Association nationale des commissions locales d'information (association loi 1901).
} 
- participation à la conférence des Présidents de CLI. Depuis la mise en place de l'ANCLI, la conférence des Présidents est coprésidée par le DGSNR et le Président de l'ANCLI ;

- organisation de sessions de formation sur le nucléaire pour les membres des CLI ;

- publication du journal DECLIC, organe de l'ANCLI, dont chaque numéro est consacré à un thème particulier, de fiches informatives à l'intention de tous les publics, d'une feuille info bimestrielle pour les CLI et des actes des colloques ${ }^{10} \ldots$

L'ANCLI a signé un protocole avec $1^{\prime} \operatorname{IRSN}^{11}$. Ce texte est important pour favoriser la circulation de l'information entre l'IRSN, les CLI et l'ANCLI. L'IRSN est, d'après son statut, l'expert public pour les problèmes nucléaires. Lorsqu'une CLI envisage une campagne d'analyse de marquage radioactif, elle est libre du choix de l'expert, mais si elle choisit l'IRSN des conditions favorables correspondant aux termes du protocole sont appliquées.

Pour aider les CLI dans les démarches à caractère scientifiques, l'ANCLI a mis en place un comité scientifique. Il se préoccupe, par exemple, du stockage du graphite résultant du démantèlement de centrale nucléaire de la filière graphite-gaz ou du refroidissement des CNPE avec l'apparition des amibes et des légionnelles. Ce comité a, malgré une grande ambition, limité son action par manque de moyens.

En plus de ces actions, l'ANCLI a noué des relations importantes pour l'avenir tant au plan national qu'international.

\section{L'évolution}

Les CLI sont mises en place par une circulaire laissant beaucoup de libertés dans les actions mais ne conférant pas de statut à ces commissions et ne prévoyant pas de financement indispensable pour des actions avec l'indépendance nécessaire à une bonne communication. L'ANCLI est une association, mais ne peut revendiquer l'adhésion de toutes les CLI.

Ces difficultés pourraient être levées lorsque la loi ${ }^{12}$ sur la transparence et la sûreté en matière nucléaire sera votée, car le projet de cette loi, entre autres, prévoit

\footnotetext{
${ }^{10}$ Les actes des colloques et des numéros de Déclic sont disponibles à l'ANCLI.

11 IRSN, Institut de radioprotection et de sûreté nucléaire.

12 Projet de loi sur la transparence et la sûreté en matière nucléaire enregistré en juin 2002 à la présidence du Sénat.
} 
de conférer le statut d'association aux CLI et définit leur financement. Cette même loi crée une fédération des CLI qui devrait remplacer l'ANCLI.

Une autre évolution concerne les sites nucléaires intéressants la Défense.

Ces sites sont exclus du champ d'application de la circulaire de 1981. Pour permettre une information des populations sans remettre en cause le secret nécessaire aux activités de ces centres, l'article 4 du décret $n^{\circ} 2001-592$ du 5 juillet 2001 relatif à la sûreté et à radioprotection des installations et activités nucléaires intéressant la défense prévoit la création de commissions d'information.

Ces commissions d'information sont créées par arrêté du Ministre de la défense ou du Ministre de l'industrie pour les sites d'exploitation des INB secrètes et ont pour mission d'informer le public des conséquences des activités nucléaires sur la santé et l'environnement.

Ce décret est une grande avancée qu'il convient de bien mettre en œuvre.

Afin d'optimiser l'information communiquée aux populations, il semble nécessaire de permettre à tous les groupes agissant au voisinage des établissements à risque et les CLI nucléaires de travailler ensemble et de participer à la même fédération. Les objets (industries chimiques, centres de traitement des déchets, ...) de ces structures sont souvent différents, mais le but communiquer et informer la population est identique.

Pour limiter les risques liés aux transports de matières nucléaires, l'arrêté ${ }^{13}$ de janvier 2004 indique que tous les transports des matières nucléaires doivent être classifiés secret défense. Une circulaire d'application précise accompagne cet arrêté, cependant cette classification peut, selon l'utilisation qui en est faite, limiter l'action des CLI. Il est nécessaire de rester vigilant afin que le rôle des CLI soit préservé.

\section{RÉFÉRENCES}

CI (1981) Circulaire du 15 décembre 1981 relative à la création de Commissions d'information auprès des grands équipements énergétiques.

Euratom (1996) Directive 96/29 du 13 mai 1996.

Métivier H. (1997) Principes et normes de radioprotection, dans Les déchets nucléaires (R. Turlay, Ed.) pp. 249-271. EDP Sciences, Les Ulis.

\footnotetext{
13 Arrêté du 26 janvier 2004 du Haut fonctionnaire de Défense auprès du Ministre de l'économie, des finances et de l'industrie, relatif à la protection du secret de la défense nationale dans le domaine de la protection et du contrôle des matières nucléaires. 